\title{
Pseudomonas Luteola Infection: First Case Report of Urinary Tract Infection and Review of Literature
}

\author{
Salma Ben Hmida ${ }^{1 \star}$, Ichrak Boughariou ${ }^{1}$, Fatma Gassara ${ }^{1}$, Majdi Maazoun ${ }^{1}$, Emna Eleuch ${ }^{1}$, Chakib Marrakchi ${ }^{1}$, \\ Dorra Lahieni ${ }^{1}$, Adnen Hammami ${ }^{2}$, Mounir Ben Jmeaa ${ }^{1}$
}

\begin{abstract}
${ }^{1}$ Département des Maladies Infectieuses, Hôpital Universitaire Hedi Chaker, Sfax, TUNISIA
${ }^{2}$ Microbiology Laboratory, Habib Bourguiba University Hospital, Sfax, TUNISIA

*Corresponding Author: salmabenhmiida@gmail.com
\end{abstract}

Citation: Ben Hmida S, Boughariou I, Gassara F, Maazoun M, Eleuch E, Marrakchi C, Lahieni D, Hammami A, Ben Jmeaa M. Pseudomonas Luteola Infection: First Case Report of Urinary Tract Infection and Review of Literature. Electron J Gen Med. 2021;18(5):em313. https://doi.org/10.29333/ejgm/11101

\begin{tabular}{|c|c|}
\hline ARTICLE INFO & ABSTRACT \\
\hline Received: 6 Mar. 2021 & 1 \\
\hline Accepted: 1 Jul. 2021 & $\begin{array}{l}\text { serious infections, mainly in immuncompromised patients. We report here the first case of urinary tract infection } \\
\text { due to } P \text {. luteola and we review, by searching in Pub Med all cases of } P \text {. luteola infection. }\end{array}$ \\
\hline
\end{tabular}

Keywords: Pseudomonas luteola, urinary tract infection, antibiotherapy

\section{INTRODUCTION}

$P$. luteola is a gram-negative non fermentative and motile bacillus [1]. It is a rare saprophyte commensal in humans, but it may cause severe infections, especially in patients with health disorders [1]. We report the first case of urinary tract infection due to $P$. luteola in a 69-year-old patient with chronic renal failure.

\section{CASE REPORT}

A 69-year-old male, with prior history of diabetes mellitus treated with insulin therapy, benign prostatic hyperplasia, and end stage renal failure requiring hemodialysis 3 times a week, presented to the hospital with dysuria, burning miction and intermittent left lumbar fossa pain for 6 days. Physical examination revealed a blood pressure of $128 / 80 \mathrm{mmHg}$, pulse of 95 beats $/ \mathrm{min}$ and body temperature of $38.7{ }^{\circ} \mathrm{C}$. The arteriovenous fistula was clean. The heart sounds were normal and there was no murmur. Abdominal examination revealed tenderness in left flank. The prostate is painless in the rectal exam. Results of Laboratory investigations included hemoglobin $(9.1 \mathrm{~g} / \mathrm{dl})$, white cells count $\left(6150 / \mathrm{mm}^{3}\right)$, C-reactive protein $(32 \mathrm{mg} / \mathrm{L})$, blood urea $(14.2 \mathrm{mmol} / \mathrm{l})$ and creatininemia (795 $\mu \mathrm{mol} / \mathrm{l})$. Abdomino-pelvic ultrasonography was normal. Urine microscopy showed countless leukocytes. The patient was treated by empirical intravenous antibiotherapy: ceftriaxone and ciprofloxacin for 2 days but no clinical improvement was noted. Blood cultures were negative. On 3th day, $P$. luteola was identified in urine. It was sensitive to piperacillin- tazobactam, ceftazidime, cefepime, aztreonam, imipenem, fosfomycin and colistin and resistant to ampicillin, augmentin, cefotaxim, ceftriaxone, norfloxacin, ciprofloxacin, gentamicin, amikacin, tobramycin, tigecycline and cotrimoxazole.

According to the results of the antibiotic susceptibility testing, ceftazidime $(1 \mathrm{~g} /$ day after dialysis) was administered parenterally. After 48 hours, the fever disappeared, urinary disorders subsided and CRP decreased. Ceftazidime was prescribed for a total duration of 11 days with good outcome. After one month, the patient was admitted again with a severe sepsis (hypotension 68/48, pulse $110 \mathrm{bpm}$ ). He has reported left flank pain with burning miction and vomiting since 6 days, without fever. Physical examination revealed apyrexia, tenderness in left lumbar fossa, and an arterial oxygen saturation equal to $94 \%$ without respiratory signs. Pulmonary auscultation was normal and the arterioveinous fistula was functional and clean. Laboratory findings included a hemoglobin of $9 \mathrm{~g} / \mathrm{dl}$, a white blood cell count of $11000 / \mathrm{mm}^{3}$ and a C-reactive protein level of $112 \mathrm{mg} / \mathrm{L}$. The serum level of urea was $28,9 \mathrm{mg} / \mathrm{dl}$ and of creatinine was $799 \mu \mathrm{mol} / \mathrm{l}$. Serum protein, bilirubin, electrolytes and liver enzyme profile were all normal. Electrocardiogram and chest X-ray were normal. His breathing and heart were monitored. At this time, the patient had urine output, so a urine sample was taken showing countless leukocytes. Considering the $P$. luteola's anterior urinary tract infection, an association of gentamicin (3 $\mathrm{mg} / \mathrm{kg} /$ day) and imipenem (500 mg/day) was started. In the following 4 hours, the patient's state worsed and he presented a cardiopulmonary arrest. The patient was dead despite resuscitative efforts. We were not able to do a post-mortem examination but we thought that our patient had presented multivisceral failure due to severe sepsis caused by urinary tract infection. Unfortunately, the urine culture returned contaminated after two days. 


\section{DISCUSSION}

P. luteola, a gram-negative aerobic bacillus, was first described by Tatum et al. and was previously known by Centers for Disease Control and Prevention (CDC) as group Ve-1 and Chryseomonas luteola [1]. Due to the close phylogenetic relatedness between Chryseomonas and Pseudomonas, this bacterium was reassigned to the genus Pseudomonas as $P$. luteola [2]. Its habitat is not determined, but it is usually found in water, soil, and moist environments [3,4]. All the previously reported cases suggest that $P$. luteola, although a rare saprophyte, could emerge as a potential pathogen [5]. The predisposing factors for infection with $P$. luteola include immunosuppressive conditions like use of corticosteroids and other immunosuppressive therapy, malignancy tumors and chronic renal failure such as our case [2]. In other cases, the infection is associated with indwelling catheters and prostheses [6,7]. Nosocomial infections are more frequent than community acquired ones, especially in immunocompromised patients [1]. In our case, the patient had a community acquired urinary tract infection by this bacterium. $P$. luteola has a variable sensitivity to penicillins, cephalosporins, tetracyclines, and cotrimoxazole and is often sensitive to imipenem, aminoglycosides and fluoroquinolones such as ciprofloxacin $[4,8,9]$. In our case, $P$. luteola was sensitive to piperacillin/ tazobactam, ceftazidime, cefepime, aztreonam and imipenem, but resistant to ampicillin, augmentin, cefotaxim, ceftriaxone, tigecycline, cotrimoxazole, amikacin, gentamicin, tobramycin and ciprofloxacin. According to our research on Pub Med from 1980 until November 2020, we found only 19 cases of $P$. luteola's infection in adults. A summary of main features of these cases is put in Table 1 [1, 4-21]. This

Table 1. Summary of all reported cases with Pseudomonas luteola's infection

\begin{tabular}{|c|c|c|c|c|c|c|}
\hline References & Infection & $\begin{array}{l}\text { Cases } \\
\text { number }\end{array}$ & Risk Factors & Susceptibility status & Treatment & Outcomes \\
\hline $\begin{array}{l}\text { Connor et al. } \\
\text { (1987) [10] }\end{array}$ & Peritonitis & 2 & $\begin{array}{l}\text { End-stage renal } \\
\text { disease }\end{array}$ & $\begin{array}{c}\text { S: tobramycin and } \\
\text { trimethoprim-sulfamethoxazole } \\
\text { R: cefazolin }\end{array}$ & $\begin{array}{c}\text { Remove dialysis catheter + } \\
\text { Antibiotherapy }\end{array}$ & Alive \\
\hline $\begin{array}{l}\text { Su et al. (2014) } \\
\text { [11] }\end{array}$ & & & Peritoneal dialysis & $\begin{array}{c}\text { S: gentamicin, amikacin, ceftazidime, ciprofloxacin, } \\
\text { imipenem, cefepime, piperacillin, and piperacillin- } \\
\text { tazobactam }\end{array}$ & $\begin{array}{c}\text { Ceftazidime + Gentamicin (15 } \\
\text { days) }\end{array}$ & Alive \\
\hline $\begin{array}{c}\text { Rastogi and } \\
\text { Sperber (1998) } \\
{[12]}\end{array}$ & & & Immunocompetent & $\begin{array}{c}\text { S: ampicillin, gentamicin, trimethoprim- } \\
\text { sulfamethoxazole, ceftriaxone, and ciprofloxacin } \\
\text { R: cefazolin and cefuroxime }\end{array}$ & $\begin{array}{l}\text { Intraveinous ceftriaxone } 2 \mathrm{~g} / \\
\text { day }\end{array}$ & Alive \\
\hline $\begin{array}{l}\text { Tsakris et al. } \\
(2002)[13]\end{array}$ & $\begin{array}{l}\text { Cutaneous } \\
\text { Infection }\end{array}$ & 6 & Sickle cell disease & $\begin{array}{l}\text { S: aminoglycosides (amikacin, gentamicin, tobramycin), } \\
\text { ciprofloxacin, ceftazidime, cefepime and imipenem } \\
\text { R: cephalosporins (cephalothin, cefuroxime, cefoxitin, } \\
\text { ceftriaxone), ampicillin, amoxycillin/clavulanate, } \\
\text { aztreonam and trimethoprim/sulfamethoxazole }\end{array}$ & $\begin{array}{l}\text { Local treatment with sterilized } \\
\text { water + local instillation of the } \\
\text { growth factor G-CSF }\end{array}$ & Alive \\
\hline $\begin{array}{l}\text { Dalamaga et al. } \\
\qquad(2004)[7]\end{array}$ & & & Steroid Therapy & $\begin{array}{l}\text { S: cefuroxime, ceftazidime, cefriaxone, cefepime, } \\
\text { aztreonam, imipenem, meropenem, quinolones } \\
\text { (ciprofloxacin, pefloxacin), } \\
\text { trimethoprime/sulfamethoxazole, aminoglycosides } \\
\text { (amikacin, gentamicin, tobramycin), ticarcillin and } \\
\text { piperacillin } \\
\text { R: ampicillin and amoxycillin/clavulanate }\end{array}$ & $\begin{array}{c}\text { Drainage of the abscess }+ \\
\text { intraveinous ceftazidime } 1 \\
\mathrm{~g}^{\star} 3 / \text { day }+ \text { intraveinous } \\
\text { amikacin } 500 \mathrm{mg}^{\star} 2 / \text { day (15 } \\
\text { Days) }\end{array}$ & Alive \\
\hline $\begin{array}{l}\text { Jayagopal et al. } \\
\text { (2004) [14] }\end{array}$ & & & Immunocompetent & S: oxytétracycline, ciprofloxacine & $\begin{array}{l}\text { Oxytetracycline followed by } \\
\text { ciprofloxacin ( } 14 \text { days) }\end{array}$ & Alive \\
\hline $\begin{array}{l}\text { Ramana et al. } \\
\text { (2010) [5] }\end{array}$ & & & $\begin{array}{l}\text { Coronary artery } \\
\text { bypass graft + high } \\
\text { blood pressure }\end{array}$ & $\begin{array}{l}\text { S: ampicillin, amoxicillinclavulinic acid, pipercillin- } \\
\text { tazobactum, gentamicin, ceftriaxone, cefotaxime, } \\
\text { ciprofloxacin, ofloxacin, imipenem, tetracycline, } \\
\text { trimethoprim-sulphamethoxazole, colistin and tigicycline }\end{array}$ & $\begin{array}{l}\text { Incision drainage and surgical } \\
\text { debridement }+ \text { amoxicillin- } \\
\text { clavulinic acid and } \\
\text { ciprofloxacin }\end{array}$ & Alive \\
\hline $\begin{array}{l}\text { Roberts et al. } \\
\text { (2018) [8] }\end{array}$ & & & Immunocompetent & $\begin{array}{c}\text { S: ceftazidime, amikacin, gentamicin and tobramycin, } \\
\text { ciprofloxacin } \\
\text { R: ampicillin, augmentin, imipenem, bactrim } \\
\text { Intermediate sensitivity: cefotaxime, ceftriaxone }\end{array}$ & Amputation + Antibiotherapy & Alive \\
\hline $\begin{array}{l}\text { Casalta et al. } \\
(2005)[15]\end{array}$ & Endocarditis & 1 & $\begin{array}{l}\text { Aortic remplacement } \\
\text { for aortic } \\
\text { insuffisancy }\end{array}$ & $\begin{array}{l}\text { S: ampicillin, ureidopenicillin, third-generation } \\
\text { cephalosporins, fluoroquinolones, aminoglycosides }\end{array}$ & $\begin{array}{l}\text { Ticarcillin + clavulanic acid } 3 \\
\mathrm{~g}^{\star} 5 / \text { day }(60 \text { days })+ \\
\text { gentamicin } 210 \mathrm{mg} \text { once a day } \\
\text { ( } 15 \text { days) }\end{array}$ & Alive \\
\hline $\begin{array}{l}\text { Goteri et al. } \\
(2010)[16]\end{array}$ & $\begin{array}{l}\text { Mediastinal } \\
\text { abscess }\end{array}$ & 1 & $\begin{array}{l}\text { Autoimmune } \\
\text { thrombocytopenia }+ \\
\text { Steroid Therapy }\end{array}$ & No data & $\begin{array}{c}\text { Meropenem } 70 \mathrm{mg} / \mathrm{kg} \text { three } \\
\text { times/day + ciprofloxacin } 500 \\
\mathrm{mg}^{\star} 2 / \text { day ( } 6 \text { weeks) }\end{array}$ & Alive \\
\hline $\begin{array}{l}\text { Anuradha et al. } \\
\text { (2010) [17] }\end{array}$ & $\begin{array}{l}\text { Biliary tract } \\
\text { infection }\end{array}$ & 1 & Immunocompetent & $\begin{array}{c}\text { S: amikacin, ciprofloxacin,imipenem, Polymyxin B } \\
\text { R: ampicillin, amoxycillin-clavulanic acid, piperacillin, } \\
\text { piperacillin-tazobactam. } \\
\text { cefotaxime, ceftriaxone, ceftazidime }\end{array}$ & $\begin{array}{c}\text { Hepaticojejunostomy }+ \\
\text { intravenous antibiotherapy } \\
\text { (cefotaxime + gentamycin }+ \\
\text { metronidazole) }\end{array}$ & Alive \\
\hline $\begin{array}{l}\text { Ngoh et al. } \\
\text { (2011) [9] }\end{array}$ & Pneumonia & 3 & $\begin{array}{l}\text { Diabetes, asthma, } \\
\text { high blood pressure, } \\
\text { inter-ventricular } \\
\text { stent }\end{array}$ & $\begin{array}{l}\text { S: piperacillin, piperacillin-tazobactam, ticarcillin, } \\
\text { ceftazidim, gentamycin, rifampicin } \\
\text { R: imipenem, aztreonam, tobramycin, amikacin, } \\
\text { ciprofloxacin, tetracyclin, and trimethoprim- } \\
\text { sulfamethoxazole }\end{array}$ & $\begin{array}{l}\text { Imipenem + amikacin + } \\
\text { teicoplanin + fuconazole }\end{array}$ & Dead \\
\hline $\begin{array}{l}\text { Jacob et al. } \\
(2015)[18]\end{array}$ & & & Immunocompetent & No data & $\begin{array}{l}\text { Intraveinous ciprofloxacin (14 } \\
\text { days) }\end{array}$ & Alive \\
\hline $\begin{array}{l}\text { Dharmayanti et } \\
\text { al. (2017) [19] }\end{array}$ & & & $\begin{array}{l}\text { Guillain Barre } \\
\text { Syndrome }\end{array}$ & No data & Intraveinous meropenem & Alive \\
\hline
\end{tabular}


Table 1 (continued). Summary of all reported cases with Pseudomonas luteola's infection

\begin{tabular}{|c|c|c|c|c|c|c|}
\hline References & Infection & $\begin{array}{c}\text { Cases } \\
\text { number }\end{array}$ & Risk Factors & Susceptibility status & Treatment & Outcomes \\
\hline $\begin{array}{c}\text { Yousefi et al. } \\
\text { (2014) [1] }\end{array}$ & $\begin{array}{l}\text { Pleuritic } \\
\text { empyema }\end{array}$ & 1 & $\begin{array}{l}\text { Pulmonary } \\
\text { tuberculosis }\end{array}$ & $\begin{array}{c}\text { S: trimethoprim-sulfamethoxazole, } \\
\text { Intermediate: gentamycin } \\
\text { R: ampicillin, cefepime, ciprofloxacin, norfloxacin, } \\
\text { nalidixicacid. }\end{array}$ & Cotrimoxazole & Alive \\
\hline $\begin{array}{l}\text { Otto et al. } \\
\text { (2013) [6] }\end{array}$ & Septicemia & 2 & Lung carcinoma & $\begin{array}{c}\text { S: third-generation cephalosporins, aminoglycosides, } \\
\text { ureidopenicillins, ciprofloxacin } \\
\text { R: first and second-generation cephalosporins }\end{array}$ & $\begin{array}{c}\text { Intraveinous piperacillin } 16 \\
\text { g/day (14 days) }\end{array}$ & Alive \\
\hline $\begin{array}{c}\text { Balew et al. } \\
(2017)[4]\end{array}$ & & & Hodgkin lymphoma & No data & No data & No data \\
\hline $\begin{array}{l}\text { Harvey et al. } \\
(2007)[20]\end{array}$ & Endophtalmitis & 2 & Immunocompetent & $\begin{array}{c}\text { S: piperacillin/tazobactam, } \\
\text { trimethoprim/sulfamethoxazole, cefepime } \\
\text { R: amikacin }\end{array}$ & $\begin{array}{c}\text { Topical medication }+ \\
\text { intravitreal injection of } \\
\text { piperacillin/tazobactam + oral } \\
\text { cotrimoxazole }\end{array}$ & Alive \\
\hline $\begin{array}{l}\text { Naik et al. } \\
(2018)[21]\end{array}$ & & & Immunocompetent & S: Ciprofloxacin & Ocular surgery + Ciprofloxacin & Alive \\
\hline \multirow[t]{2}{*}{$\begin{array}{l}\text { Our case, } \\
2020\end{array}$} & $\begin{array}{l}\text { Urinary tract } \\
\text { infection }\end{array}$ & 1 & $\begin{array}{l}\text { End-stage renal } \\
\text { disease }\end{array}$ & $\begin{array}{l}\text { S: piperacillin-tazobactam, ceftazidime, cefepime, } \\
\text { aztreonam, imipenem, fosfomycin, colistin } \\
\text { R: ampicillin, augmentin, cefotaxim, ceftriaxone, } \\
\text { ciprofloxacin, gentamicin, amikacin, tobramycin, } \\
\text { tigecycline and cotrimoxazole. }\end{array}$ & $\begin{array}{l}\text { First episode : ceftazidime (1 } \mathrm{g} \\
\text { after dialysis), } 11 \text { days }\end{array}$ & Recovery \\
\hline & & & & & $\begin{array}{l}\text { Second episode : gentamicin ( } 3 \\
\mathrm{mg} / \mathrm{kg} / \text { day) + imipenem ( } 500 \\
\mathrm{mg} / \text { day })\end{array}$ & Dead \\
\hline
\end{tabular}

microorganism was reported to produce septicemia [4-7,9,12], endocarditis [15], pleuritic empyema [1], mediastinal abscess $[16]$, pneumonia $[9,18,19]$, peritonitis $[10,11]$, biliary tract infection [17], endophtalmitis $[20,21]$ and cutaneous infection $[5,7,8,12,14]$. To the best of our knowledge, our case is the first reported case of urinary tract infection caused by $P$. luteola. The majority of cases in the literature review (15/20) had progressed favourably under adequate antibiotic treatment. Ngoh et al. [9] reported a fatal case of $P$. luteola's pneumonia in a patient with multiple comorbidities. The patient was admitted to the Intensive Care Unit and he had received an aggressive therapy (imipenem, amikacin, teicoplanin, and fluconazole) but he was died three days later after multivisceral failure. The strain was resistant to imipenem, and amikacin. Our patient had two episodes of UTI one month apart. In the first one, $P$. luteola was identified and the patient was treated by intravenous ceftazidime with a clinical improvement. In the second one, the patient received an association of gentamicin and imipenem but his clinical state worsed and he was dead after 4 hours of intensive care. We had no idea about the microorganism implicated in this recurent UTI but $P$. luteola can be the incriminated agent, in particular a strain resistant to imipenem and aminoglycosides. Due to its variable resistance pattern, $P$. luteola should be considered as a possible culprit when infections are unresolving, particularly in immunocompromised patients [8].

\section{CONCLUSION}

In this work we have reported on the main aspects of $P$. luteola infections in adults. Due to the gravity associated with infection by $P$. luteola in such cases, we believe it is useful to report on our experience for the purpose of increasing knowledge in P. luteola and its pathological complications and improving the treatment of this infection especially for those already infected.

Author contributions: All authors have sufficiently contributed to the study, and agreed with the results and conclusions.
Funding: No funding source is reported for this study. Declaration of interest: No conflict of interest is declared by authors.

\section{REFERENCES}

1. Yousefi F, Shoja S, Honarvar N. Empyema Caused by Pseudomonas luteola: A Case Report. Jundishapur J Microbiol. 2014; 7(7):e10923. https://doi.org/10.5812/ jjm.10923 PMid:25368791 PMCid:PMC4216571

2. Chihab W, Alaoui AS, Amar M. Chryseomonas luteola Identified as the Source of Serious Infections in a Moroccan University Hospital. J Clin Microbiol. 2004; 42(4):1837-9. https://doi.org/10.1128/JCM.42.4.1837-1839.2004 PMid:15071064 PMCid:PMC387548

3. Doublet B, Robin F, Casin I, Fabre L, Le Fleche A, Bonnet R. Molecular and biochemical characterization of the natural chromosome-encoded class A beta-lactamase from Pseudomonas luteola. Antimicrob Agents Chemother. 2010; 54(1):45-51. https://doi.org/10.1128/AAC.00427-09 PMid:19884377 PMCid:PMC2798517

4. Balew A, Yimtubezinash W-A, Kelemework A, Ezra B, Abdulaziz A, Daniel A. Rare bacterial isolates causing bloodstream infections in Ethiopian patients with cancer. Infect Agent Cancer. 2017; 12:40. https://doi.org/10.1186/ s13027-017-0150-9 PMid:28702079 PMCid:PMC5504797

5. Ramana KV, Kareem MA, Sarada CHV, Sebastian S, Lebaka $\mathrm{R}$, Ratnamani MS, et al. Chryseomonas luteola bacteremia in a patient with left pyocele testis with Fournier's scrotal gangrene. Indian J Pathol Microbiol. 2010; 53(3):568. https://doi.org/10.4103/0377-4929.68280 PMid:20699531

6. Otto MP, Foucher B, Dardare E, Gerome P. Severe catheter related bacteremia due to Pseudomonas luteola. Med Mal Infect. 2013; 43:170-1. https://doi.org/10.1016/ j.medmal.2013.01.013 PMid:23473706

7. Dalamaga M, Karmaniolas K, Chavelas C, Liatis S, Matekovits $\mathrm{H}$, Migdalis I. Pseudomonas luteola cutaneous abscess and bacteraemia in a previously healthy man. Scand J Infect Dis. 2004; 36(6-7):495-7. https://doi.org/ 10.1080/00365540310016196 PMid:15307579 
8. Roberts W, Roessler C, Francis PJ, Noel D, Loukas M. Postsurgical Gangrene with Pseudomonas luteola Resulting in Limb Amputation: A Case Review. Cureus. 2018;10(10):e3441. https://doi.org/10.7759/cureus.3441

9. Ngoh E, Zohoun A, Ghazouani M, El Hamzaoui S, El Ouennass M. A fatal case of Pseudomonas luteola infection. Med Mal Infect. 2011; 41(7):399-400. https://doi.org/ 10.1016/j.medmal.2010.12.018 PMid:21439742

10. Connor BJ, Kopecky RT, Frymoyer PA, Forbes BA. Recurrent Pseudomonas luteola (CDC group Ve-1) peritonitis in a patient undergoing continuous ambulatory peritoneal dialysis. J Clin Microbiol 1987;25:1113-14. https://doi.org/ 10.1128/jcm.25.6.1113-1114.1987 PMid:3597754

11. Su SY, Chao CM, Lai CC. Peritoneal dialysis peritonitis caused by Pseudomonas luteola. Perit Dial Int J Int Soc Perit Dial. 2014;34(1):138-9. https://doi.org/10.3747/ pdi.2012.00265 PMid:24525608 PMCid:PMC3923710

12. Rastogi S, Sperber SJ. Facial cellulitis and Pseudomonas luteola bacteremia in an otherwise healthy patient. Diagn Microbiol Infect Dis. 1998;32:303-5. https://doi.org/10.1016 /S0732-8893(98)00082-0

13. Tsakris A, Hassapopoulou H, Skoura L, Pournaras S, Douboyas J. Leg ulcer due to Pseudomonas luteola in a patient with sickle cell disease. Diagn Microbiol Infect. 2002; 42:141-3. https://doi.org/10.1016/S0732-8893(01) 00336-4

14. Jayagopal S, Berry MG, Ross G, Howcroft AJ. Hand infection caused by Chryseomonas luteola. Br J Plast Surg. 2004; 57(7):694-5. https://doi.org/10.1016/j.bjps.2004.05.028 PMid:15380711

15. Casalta, J.P, Fournier, P.E, Habib, G, Riberi, A, Raoult, D. Prosthetic valve endocarditis caused by Pseudomonas luteola. BMC Infect Dis 2005; 5: 82. https://doi.org/ 10.1186/1471-2334-5-82 PMid:16221303 PMCid: PMC1274313
16. Goteri G, Dorrestein GM, Manso E, Giantomassi F, Rubini C, Zizzi A. Chryseomonas luteola: an unusual clinical infection mimicking a mediastinal malignant lymphoma. Pathol Lab Med Int. 2010; 2:137-9. https://doi.org/10.2147/ PLMI.S13645

17. Anuradha S De, Salunke PP, Parikh HR, Baveja SM. Chryseomonas luteola from Bile Culture in an adult male with sever jaundice. J Lab Physicians 2010; 2:40-1. https://doi.org/10.4103/0974-2727.66708 PMid:21814406 PMCid:PMC3147085

18. Jacob N, Weeraporn S, Shannon Y. Round pneumonia with Pseudomonas luteola and Escherichia vulneris bacteremia. The Southwest Respiratory and Critical Care Chronicles. 2015; 3 (12). https://doi.org/10.12746/swrccc2015.0312.153

19. Dharmayanti A, Astrawinata D. Ventilator-Associated Pneumonia (VAP) in a Patient with Guillain-Barre Syndrome. Acta Medica Indones. 2017; 49 (2):151.

20. Uy Harvey.S, Leuenberger E.U, de Guzman, B.B, Natividad, F.F. Chronic, postoperative Pseudomonas luteola endophthalmitis. Ocul Immunol Inflamm 2007; 15: 359-61. https://doi.org/10.1080/09273940701396697 PMid:17763136

21. Naik AU, Prakash VJ, Susvar P, Therese KL, Parameswari CK. Postoperative endophthalmitis due to Pseudomonas luteola: First reported case of acute and virulent presentation from a tertiary eye care center in South India. Indian J Ophthalmol. 2018; 66(8):1200. https://doi.org/ 10.4103/ijo.IJO_242_18 PMid:30038182 PMCid: PMC6080441 